\title{
Impact of Pregnancy on the Sex Life of Women: State of the Art
}

\author{
Liliam Renata Silveira Santiago, Lucia Alves da Silva Lara*, \\ Adriana Peterson Mariano Salata Romão, Maria Fernanda Barbirato da Mata Tiezzi, \\ Ana Carolina Japur de Sá Rosa e Silva
}

Department of Gynecology and Obstetrics, Faculty of Medicine of Ribeirão Preto, University of São Paulo, Ribeirão Preto, Brazil. Email: *luciaalvess@yahoo.com.br

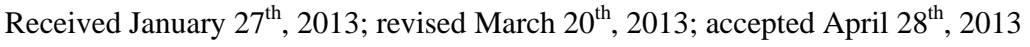

Copyright (c) 2013 Liliam Renata Silveira Santiago et al. This is an open access article distributed under the Creative Commons Attribution License, which permits unrestricted use, distribution, and reproduction in any medium, provided the original work is properly cited.

\begin{abstract}
Introduction: Women's sexual function is a complex ensemble of variables that involve physical, emotional, and psychosocial states. Objective: This review aims to evaluate the evidence about the sexual behavior of women during pregnancy. Methods: Survey of PubMed for the period from 1996 to 2011 for prospective, retrospective and case-control studies, randomized clinical trials, meta-analyses and systematic reviews on the sexual function of women during pregnancy using the terms “sexual function”, “sexual dysfunction”, “sexuality”, "pregnancy”, and "pregnant woman”. Results: Changes occur in the sexual function of women during pregnancy; however, due to the heterogeneity of the studies and the incomparability of samples for lack of pairing for cultural, age, length of relationship and gestational age variables, and other methodological biases, it is not possible to characterize the sexual response of pregnant women. Conclusions: The changes observed are not sufficient to characterize sexual behavior during pregnancy as pathological. It is therefore necessary to standardize the study groups and the instruments for the assessment of sexual function, to determine the presence of distress concomitant with changes in sexual function, and to assess the sexual function of the partner and the impact of changes on the dyadic relationship before and after the gestational period.
\end{abstract}

Keywords: Sexual Function; Dysfunction; Sexuality; Pregnancy; Pregnant

\section{Introduction}

The last few decades have been marked by particular scientific interest in female sexual behavior [1], especially in situations such as the gestational period, which can course with an increased prevalence of sexual dysfunction $[1,2]$.

The nature of female sexuality is complex and contextual and is correlated with mental health [3] and feelings for the partner $[4,5]$, as well as with other factors that may modify the physical and psychological condition of a woman [2]. In general population, $40 \%$ to $50 \%$ of women may experience some form of sexual dysfunction during their lifetime [6].

During the gestational period, in addition to the presence of a great hormonal impact, emotional changes may occur in life style and self-image, possibly changing the expression of sexuality and sexual behavior [7-9].

\footnotetext{
"Corresponding author.
}

During the first trimester of pregnancy, progesterone is responsible for the physical and mental changes that may interfere with sexual functioning of the pregnant women. In addition, progesterone increases the sensitivity and excitability of the breasts, and promotes congestion of vulvar and vaginal tissue which may lead to dyspareunia, and provoke increased vaginal flow and involuntary urine loss, conditions that may negatively affect sex life [10]. In addition, the hormone induces vasodilation and, in consequence loss of pressure levels can occur, possibly causing fatigue and the emotional changes frequently related to depressive states [10].

Psychological changes, such as emotional lability with a tendency to depression, reduced self-esteem and the feeling of being less attractive [11] are common conditions among pregnant women, which can also interfere with sexual response. This concern with body pattern was demonstrated in a recent study showing that body image is a component of satisfaction and of the feeling of 
comfort with one's body in front of one's partner [12].

Cultural differences and myths about sexual practice are also other factors that contribute to altering the sexual response during pregnancy [13]. The orgasm, for example, courses with an oxytocin peak that stimulates contraction of the uterine fibers [14] which, although not sufficient to induce labor in a pregnancy of habitual risk, may generate discomfort and insecurity for the couple. Several studies have included fear of damage the fetus and of postcoital bleeding as factors associated with reduced sexual frequency [7,15-18]. These myths typical of each culture may also be responsible for the diverse sexual behavior of pregnant women.

A survey of the literature showed that changes appear to occur in the sexuality of pregnant women, although there is no consensus about this aspect. On this basis, the objective of the present study was to determine the possible modifications of sexual behavior in women during the gestational period.

\section{Methods}

The present literature review was based on the survey of the PubMed data base regarding studies on the sex function of women during pregnancy published from January 1966 to December 2011. The search involved prospective, retrospective and case-control studies, randomized clinical studies, meta-analyses, and systematic reviews. To this end, the following terms were used: "sexual function", "sexual dysfunction", "sexuality and pregnancy" and "pregnant woman". The survey was limited to studies conducted on humans and published in English, which used instruments and/or structured questionnaires to determine the sexual function of pregnant women. Studies that performed only qualitative analyses were excluded. A total of 1055 articles were selected. Two investigators selected the studies in an independent manner. After refinement, 24 articles that satisfied the inclusion criteria were left, and are listed in Table 1.

Cross-sectional and cohort studies were included for the evaluation of sexual function and reviews and metaanalyses were considered to compare the data of the present study.

\section{Results}

Twenty-four articles were included, 14 of them crosssectional studies, five cohort studies, one a clinical trial, three reviews, and one a meta-analysis. The Female Sexual Function Index (FSFI) was the instrument used in seven studies and was the only one used for of evaluation of sexual function in four of them, whereas it was associated with other instruments in the other three. It is interesting to note the variability of the cut-off points of the FSFI score used by the several authors. A cut-off from
26.55 to 30 was considered to indicate a risk for sexual function. The seven studies that used the FSFI revealed that sexual function declines during the gestational period, with the third trimester presenting the lowest FSFI scores regarding the cut-off points used. Only one study reported greater sexual satisfaction during the gestational period [15,19-24].

Four studies evaluated sexual behavior using a modified version of the Pregnancy and Sexuality Questionnaire (PSQ). In a study with 141 pregnant women, Bartellas et al. [7] detected orgastic dysfunction in 34\% of them. Fantasy was referred by $44 \%$ of the sample, $97 \%$ referred kissing, and 31\% masturbation. In $79 \%$ of the couple sexual games was part of sexual behavior and caresses of the breast in $87 \%$, and vaginal coitus in $86 \%$ of the women interviewed. Fok et al. [17], using a modified version of the PSQ, demonstrated an overall reduction of sexual activity in $93 \%$ of pregnant women, especially during the third trimester, although sexual games represented by breasts caresses and kissing were clearly present. A retrospective Brazilian study conducted during the immediate puerperium [25] detected higher PSQ scores for sexual desire during the third trimester among women who took the initiative in sex.

Nine studies used structured questionnaires for the evaluation of sexual function. Sexual desire remained unchanged in $60 \%$ of 500 Nigerian women who were in different gestational periods; however, coital frequency was reduced in $64 \%$ of them due to nausea and vomiting, fear of abortion, fear of fetal damage, physical limitation, lack of interest, and discomfort [26]. Frequency was unchanged in $32 \%$ of them, who considered sexual relations to be important for maintaining the marital relationship and harmony. This reason was also reported by $26 \%$ of 375 pregnant women studied by Bello et al. [27]. In a study of 360 Asian women, Senkumwong et al. [9] detected reduction of sexual desire with the advent of pregnancy. In the cited study, $79.7 \%$ of the pregnant women reported fear of sexual practice and of damage to the fetus. In a retrospective evaluation of puerperae performed on the day of hospital discharge, Pauleta et al. [18] observed that the first trimester was the one of highest frequency of sexual relations (44.7\%) and that $55 \%$ reported a reduction of sexual frequency during the third trimester. Fear of sexual relations was reported by $23.4 \%$ of the women and sexual desire was reduced in $32.5 \%$ of them. A perception of lower attractiveness was reported by $41.5 \%$ and reduced sexual interest of the partner was reported by $24.5 \%$. Sexual satisfaction was unchanged in $48.4 \%$ of the women. Eryilmaz et al. [16] evaluated the perception of 238 women regarding their sex life before and during pregnancy. Eighty percent of the pregnant women reported worsening of sexual function and $61 \%$ felt that sexual relations represented a risk 
Table 1. Summary of the studies surveyed.

\begin{tabular}{|c|c|c|c|c|}
\hline Author/Year & Design & Tool & $\mathbf{N}$ & Main results \\
\hline Aslan, Aslan et al., 2005 & Prospective & FSFI & 40 & $\begin{array}{l}\text { Sexual function worsens significantly } \\
\text { throughout pregnancy. }\end{array}$ \\
\hline Bartellas, Crane et al., 2000 & Cross-sectional & PSQ, modified & 141 & $\begin{array}{l}\text { Sexual activity is reduced throughout } \\
\text { pregnancy, } 58 \% \text { of the pregnant women have } \\
\text { reduced desire, and } 49 \% \text { fear damage to the } \\
\text { fetus. }\end{array}$ \\
\hline Bello, Olayemi et al., 2011 & Cross-sectional & $\begin{array}{c}\text { Structured } \\
\text { questionnaire }\end{array}$ & 375 & $\begin{array}{l}\text { Desire increases but the frequency of orgasms } \\
\text { decreases. Marriage and cohabiting were } \\
\text { predictors of sex and pain and extramarital } \\
\text { experience was an impediment for sex. }\end{array}$ \\
\hline Chang, C. et al., 2010 & Cross-sectional & FSFI & 663 & $\begin{array}{l}\text { Total FSFI scores, frequency and satisfaction } \\
\text { differ significantly throughout pregnancy, } \\
\text { while desire score does not change. }\end{array}$ \\
\hline Dejudicibus and McCabe, 2002 & Prospective & $\begin{array}{c}\text { Structured } \\
\text { questionnaire }\end{array}$ & 138 & $\begin{array}{l}\text { Marital satisfaction explained better rates of } \\
\text { sexual satisfaction during pregnancy and was a } \\
\text { predictor of sexual desire and satisfaction. } \\
\text { Depression was an important predictor of } \\
\text { reduced desire during pregnancy. }\end{array}$ \\
\hline Erol, B. et al., 2007 & Cross-sectional & FSFI & 589 & $\begin{array}{l}\text { The FSFI score is significantly lower in the } \\
\text { third trimester. The most common sexual } \\
\text { dysfunction is the reduced clitoral sensitivity, } \\
\text { followed by reduced sexual desire. }\end{array}$ \\
\hline Eryilmaz, Ege et al., 2004 & Cross-sectional & $\begin{array}{c}\text { Structured } \\
\text { questionnaire }\end{array}$ & 238 & $\begin{array}{l}61.4 \% \text { fear fetal damage, } 81.5 \% \text { have their sex } \\
\text { life affected during pregnancy as a } \\
\text { consequence of exhaustion, fatigue and } \\
\text { reduced desire. There was a significant } \\
\text { negative relation between sex life and } \\
\text { duration of marriage. }\end{array}$ \\
\hline Fok, Chan et al., 2005 & Cross-sectional & PSQ, modified & 298 & $\begin{array}{l}93 \% \text { reported reduced sexual activity, } 60 \% \text { of } \\
\text { the women and } 40 \% \text { the men reported reduced } \\
\text { sexual desire and pleasure, and } 80 \% \text { of the } \\
\text { couples worried about the risk of sexual } \\
\text { activity for the fetus. }\end{array}$ \\
\hline Gokyildiz and Beji, 2005 & Cross-sectional & $\begin{array}{c}\text { Structured } \\
\text { questionnaire }\end{array}$ & 150 & $\begin{array}{l}\text { Coital frequency was reduced during } \\
\text { pregnancy and the time of sexual intercourse } \\
\text { decreased with the course of pregnancy. }\end{array}$ \\
\hline Johnson, C. E. 2011 & Review & & & $\begin{array}{l}\text { Despite the fears and myths regarding sexual } \\
\text { activity during pregnancy, maintaining the } \\
\text { couple's sexual interaction can promote sexual } \\
\text { health and marital proximity. }\end{array}$ \\
\hline Kamis, Mustafa et al., 2007 & Cross-sectional & $\begin{array}{c}\text { Structured } \\
\text { questionnaire }\end{array}$ & 190 & $\begin{array}{l}77 \text { have a reduced sexual desire during } \\
\text { pregnancy and } 43.7 \% \text { believe that sexual } \\
\text { intercourse may cause fetal damage. }\end{array}$ \\
\hline kerdarunsuksri \& Manusirivithaya, 2010 & Cross-sectional & FSFI & 347 & $\begin{array}{l}\text { There was a significant reduction in all FSFI } \\
\text { domains, with } 93.4 \% \text { presenting a score of less } \\
\text { than } 26.5 \% \text {. }\end{array}$ \\
\hline Leite, Campos et al., 2009 & Prospective & FSFI & 271 & $\begin{array}{l}\text { FSFI was similar in the first two trimesters and } \\
\text { became significantly worse in the third. This } \\
\text { impact was lower among adolescent pregnant } \\
\text { girls. }\end{array}$ \\
\hline Murtagh, J., 2010 & Review & FSFI & & $\begin{array}{l}\text { Female sexual function requires consistent } \\
\text { tools for better evaluation. It is necessary to } \\
\text { improve counseling during the prenatal visits. }\end{array}$ \\
\hline
\end{tabular}


Naldoni, Pazmini et al., 2011

Cross-sectional

FSFI

Orji, Ogunlola et al., 2002

Pauleta. Pereira et al., 2010

Pauls, Occhino et al., 2008

Sacomori and Cardoso, 2010

Senkuwong, Chaovisitsaree et al., 2006

Serati, M., 2010

Uwapusitanon and Choobun, 2004

von Sydow, K., 1999

Wannakosit and Phupong, 2010
Clinical FSFItrial
Structured

Cross-sectional

Cross-sectional

Prospective

Cross-sectional

Cross-sectional

Review

Prospective

Structured questionnaire

Meta-analysis

Structured questionnaire

FSFI

PSQ

Structured questionnaire

pregnancy, no change in orgasmic capacity , $79.7 \%$ fear fetal damage,

Overall sexual function worsened significantly throughout pregnancy, especially during the third trimester.

Sexual desire, frequency, excitement, orgasm, and pleasure were reduced.

On average, female sexual desire and coital frequency are discretely reduced during the first trimester, show variable patterns in the second, and are drastically reduced in the third trimester. Data about the fathers and sexual feelings were scarce.

Desire, excitement, satisfaction and orgasm did not change in the 2 groups (with and without an educational action about sexuality), and there was a reduction of coital frequency in more than $90 \%$ of the pregnant women in the 2 groups. for the fetus. The main reasons for the decline were fatigue and exhaustion and reduced desire. The time of the relationship, as well as schooling and parity, showed a significant negative correlation with the change in sexual behavior.

Kamis et al. [13] studied 190 pregnant women during different gestational periods and non-pregnant women who had had at least one gestation, aged 17 to 49 years, and detected an increase in sexual desire in $37.4 \%$ of them. Forty-eight percent reported improved sexual satisfaction during the second trimester and $43.7 \%$ believed that sexual relations can affect gestation [13]. Uwapusitanon and Choobun [13] prospectively evaluated 149 pregnant women using a structured questionnaire and observed that desire, excitement, frequency, orgasm and satisfaction decreased significantly throughout pregnancy [13]. Dejudicibus \& McCabe [28] studied 104 primigravidae aged 22 - 40 years and detected reduction of sexual desire, frequency and satisfaction. Depression was a predictor of reduced sexual desire and satisfaction and a better mood was associated with better rates of sexual satisfaction. According to Gokyildiz and Beji [29], the frequency and time of intercourse and sexual satisfaction decrease with increasing gestational age. In this group, $33 \%$ of the pregnant women studied in the in the third trimester reported signs of marital dissatisfaction of the 
partner.

\section{Discussion}

The present review demonstrates that the most recent studies are in contrast to the first literature reports regarding the expression of sexuality and the quality of female sexual function during the gestational period. According to the work of Masters and Jonhson [30], sexual behavior of pregnant women remains unchanged during the first trimester of gestation, with improved sexual function occurring during the second trimester.

A survey of the literature revealed a wide methodological variety regarding the topic since the instruments were not always validated and most studies lacked control of important variables such as culture, age, relational aspects, and partner's sexual function, among others that may interfere with the results. Such problems may prevent studies to establish the real impact of pregnancy on women sexual functioning as well as couple's sexual functioning [31]. Even so, the data show symmetry favoring a modification not only of the sexual response, but also of the sexual behavior of pregnant women that may be related to biopsychic changes commonly occurring during this period.

An abrupt change occurs in the hormonal profile of pregnant women, with an increase of prolactin levels, which acts negatively on sexual desire [14,32]. On the other hand the increase in circulating estrogen and androgen [33], which is known to modulate sexual response also occur. Additionaly, the change of sexual behavior during pregnancy seems to be also influenced by the time of relationship of the couple, as well as culture aspects, age, physical changes and discomfort related to this period [7], and by the fear of fetal damage [16].

According to Johnson's study [18], changes in sexual function during pregnancy do not only affect couple's individually. Instead it may also have a negative influence on partner [34], by reducing his sexual interest and therefore affecting the interaction of the couple. This condition worsens according to the quality of the relationship, whereby marital adjustment and stability, commitment and communication between partners favor a good sexual relationship [12]. In 2003, Bancroft et al. [5] reported that the main predictor of sexual problems is not linked to the physiology of the sexual response cycle, but rather to the emotional and marital satisfaction.

Age seems to be an important predictor of changes of sexual function among pregnant women, being more evident among adult women [15,22,29]. Indeed, according to Basson [35], age is one of the factors involved in sexual dysfunction. However, this is still a controversial topic since a study conducted in Turkey demonstrated that age does not change sexual behavior [16]. This disagree- ment, however, may express the cultural difference involved in sexual behavior in different countries. Within this context, a Nigerian study demonstrated that coital frequency did not change in 32\% of pregnant women and increased in $4 \%$, the main reason being the belief that this behavior maintains marital harmony [26]. This result was reproduced in another Nigerian study which also detected an increased sexual desire [27]. Also regarding cultural aspects, Chinese women did not show changes in sexual desire [17] and women that had negative body image before pregnancy improved their self-perception with the progression of gestational age [20]. Previous studies had already reported that body image is a predictor of sexual satisfaction in women [12] and that sexual dysfunction is associated with a negative body image [24], with appearance being an important component of the human sexual experience [36].

According to most authors, sexual function worsens throughout pregnancy [15,19,20,23,24], with desire being the most affected phase of the sexual response [7-9, $16,17,21,26,27]$. A perceived tendency toward improvement of the sexual pattern occurs during the second trimester. This aspect was observed in a Brazilian study and seems to be possibly associated with the improvement of the symptoms occurring in the first trimester [22]. In fact, stratification of the studies reveals that the first trimester is characterized by reduced coital frequency explained by nausea, vomiting and fear of bleeding [13,16, 26,27,37]. According to Kitzinger [38], the recovery of sexual function during the second trimester is due to the reduction of physical discomfort and of the fear of fetal damage experienced at the beginning of pregnancy, as well as to the increased vaginal flow which may result in greater facility in reaching orgasm [38]. These data were reproduced in other studies which demonstrated a recovery of the sexual pattern in the second trimester $[13,20$, $22,34,39]$. This is also explained by the fact that this is the gestational period associated to the best emotional condition due to the greater stability of pregnancy [22]. Nevertheless, two studies reported that sexual satisfaction is greater during the third trimester justified by the perception of greater attractiveness of the pregnant woman during this period $[13,17]$. However, the authors included women who were pregnant at the time of interview or had been pregnant before, and assessed women sexual functioning by using a non-validated questionnaire. Such methodological bias was also detected in other studies reviewed in the present work.

It is worth to point out that methodological limitations and biases existing in the literature may characterize the sexual changes of gestation as dysfunctions instead of considering the possibility that this behavior may simply express the appropriate and physiological changes that occur in most women [34]. Although pregnancy causes 
changes in the female sexual behavior, it is not clear whether this represents sexual dysfunction. It is important to emphasize that a diagnosis of sexual dysfunction is based on the presence of suffering of the individual with respect to his/her sexual complaint [40]. Two studies demonstrated the absence of suffering with the reduction of sexual desire [15] and satisfaction with emotional closeness [23] despite the mean FSFI score at risk for sexual dysfunction. Indeed, for many couples the birth of the child is a time of joy that renders them more susceptible to greater emotional proximity [28]. For some women, marital satisfaction has a positive influence on sexual desire, with those showing better rates of sexual satisfaction having a lower decline of desire and a higher coital frequency during pregnancy [28].

An important result observed is that the literature lacks evidence about the impact of pregnancy on the sexual function of the partner. In the present review, only two studies evaluated this aspect, demonstrating that $40 \%$ of the men present reduced desire and sexual satisfaction [17] and $26.1 \%$ avoid sexual activity during the gestational period [16] for fear or due to the reduced attractiveness of the woman.

In view of the above considerations, it is clear that controlled studies are needed to assess the true impact of pregnancy on the sexual behavior of pregnant women. In order to contextualize sexual behavior during pregnancy it is mainly necessary to evaluate dyadic relationship. There are frequent reports of the importance of this aspect both regarding the safety of the practice during a pregnancy of habitual risk and regarding the preparation of couples about the changes expected during this period in order to promote marital health and well-being. Nevertheless, the lack of professional counseling to pregnant women about their sexual life has been extensively reported $[7,39,41]$. Health professional may avoid tell women about this subject possible due to lack of knowledge about sexuality. According to Kingsberg et al. [42], both the lack of time and the formality involved in approaching this subject can impair a full assessment of sexual health. It is known that a considerable number of women would like to discuss sexuality with their doctors, but $68 \%$ of them do not express this wish for fear of causing embarrassment to the professional [43]. However, a study evaluated the impact of educational intervention for pregnant women and did not detect a difference between the study and control groups. The limitation of that study was that the educational action occurred only once and only for a period of 20 minutes [37].

Thus, the difficulty of the professionals involved in prenatal care in approaching sexual function [17] frustrates the expectations of the women and fails to consider the excellent opportunity of promoting sexual health during this important period of life.
In conclusion, changes in female sex function occur during the gestational period. However, due to the heterogeneity of the studies and the impossibility of comparing samples because of lack of pairing in terms of culture aspects, age range, time of relationship and gestational age, in addition to other methodological biases, it is not possible to characterize the sexual response of pregnant women. The changes observed are not sufficient to characterize the sexual behavior during pregnancy as pathological. Thus, it is necessary to standardize and instruments for the assessment of sex function, to determine the presence of distress concomitant with changes in sex function, to assess the partner's sexual function and the impact of the changes on the dyadic relationship before and after the gestational period.

\section{REFERENCES}

[1] C. V. Rudge, et al., "Design and Validity of a Questionnaire to Assess Sexuality in Pregnant Women,” Reproductive Health, Vol. 6, 2009, p. 12. doi:10.1186/1742-4755-6-12

[2] J. Murtagh, "Female Sexual Function, Dysfunction, and Pregnancy: Implications for Practice," Journal of Midwifery \& Women's Health, Vol. 55, No. 5, 2010, pp. 438446. doi:10.1016/j.jmwh.2009.12.006

[3] U. Hartmann, et al., "Female Sexual Desire Disorders: Subtypes, Classification, Personality Factors and New Directions for Treatment," World Journal of Urology, Vol. 20, No. 2, 2002, pp. 79-88. doi:10.1007/s00345-002-0280-5

[4] P. J. Nobre, et al., "Determinants of Sexual Arousal and Accuracy of Its Self-Estimation in Sexually Functional Males," The Journal of Sex Research, Vol. 41, No. 4, 2004, pp. 363-371. doi:10.1080/00224490409552243

[5] J. Bancroft, J. Loftus and J. S. Long, "Distress about Sex: A National Survey of Women in Heterosexual Relationships,” Archives of Sexual Behavior, Vol. 32, No. 3, 2003, pp. 193-208. doi:10.1023/A:1023420431760

[6] E. O. Laumann, A. Paik and R. C. Rosen, "Sexual Dysfunction in the United States: Prevalence and Predictors," Journal of the American Medical Association, Vol. 281, No. 6, 1999, pp. 537-544. doi:10.1001/jama.281.6.537

[7] E. Bartellas, et al., "Sexuality and Sexual Activity in Pregnancy,” British Journal of Obstetrics \& Gynecology, Vol. 107, No. 8, 2000, pp. 964-968. doi:10.1111/j.1471-0528.2000.tb10397.x

[8] W. Uwapusitanon and T. Choobun, "Sexuality and Sexual Activity in Pregnancy," Journal of the Medical Association of Thailand, Vol. 87, No. S3, 2004, pp. S45-S49.

[9] N. Senkumwong, et al., "The Changes of Sexuality in Thai Women during Pregnancy,” Journal of the Medical Association of Thailand, Vol. 89, No. S4, 2006, pp. S124S129.

[10] H. Brtnicka, P. Weiss and J. Zverina, "Human Sexuality during Pregnancy and the Postpartum Period," Bratislavské Lekárske Listy, Vol. 110, No. 7, 2009, pp. 427-431. 
[11] R. R. Alteneder and D. Hartzell, “Addressing Couples' Sexuality Concerns during the Childbearing Period: Use of the PLISSIT Model,” Journal of Obstetric, Gynecologic, \& Neonatal Nursing, Vol. 26, No. 6, 1997, pp. 651658. doi:10.1111/j.1552-6909.1997.tb02739.x

[12] Y. Pujols, B. N. Seal and C. M. Meston, "The Association between Sexual Satisfaction and Body Image in Women," The Journal of Sexual Medicine, Vol. 7, No. 2, 2010, pp. 905-916. doi:10.1111/j.1743-6109.2009.01604.x

[13] M. A. Khamis, et al., "Influence of Gestational Period on Sexual Behavior," The Journal of the Egyptian Public Health Association, Vol. 82, No. 1-2, 2007, pp. 65-90.

[14] M. Fava, et al., "Psychosomatic Aspects of Hyperprolactinemia," Psychotherapy and Psychosomatics, Vol. 40, No. 1-4, 1983, pp. 257-262. doi:10.1159/000287773

[15] A. Kerdarunsuksri and S. Manusirivithaya, "Attitudes and Sexual Function in Thai Pregnant Women," Journal of the Medical Association of Thailand, Vol. 93, No. 3, 2010, pp. 265-271.

[16] G. Eryilmaz, E. Ege and H. Zincir, "Factors Affecting Sexual Life during Pregnancy in Eastern Turkey,” Gynecologic and Obstetric Investigation, Vol. 57, No. 2, 2004, pp. 103-108. doi:10.1159/000075582

[17] W. Y. Fok, L. Y. Chan and P. M. Yuen, "Sexual Behavior and Activity in Chinese Pregnant Women," Acta Obstetricia et Gynecologica Scandinavica, Vol. 84, No. 10, 2005, pp. 934-938.

[18] J. R. Pauleta, N. M. Pereira and L. M. Graca, "Sexuality during Pregnancy,” The Journal of Sexual Medicine, Vol. 7, No. 1, 2010, pp. 136-142. doi:10.1111/j.1743-6109.2009.01538.x

[19] G. Aslan, et al., "A Prospective Analysis of Sexual Functions during Pregnancy," International Journal of Impotence Research, Vol. 17, No. 2, 2005, pp. 154-157. doi:10.1038/sj.ijir.3901288

[20] S. R. Chang, et al., "Comparison of Overall Sexual Function, Sexual Intercourse/Activity, Sexual Satisfaction, and Sexual Desire during the Three Trimesters of Pregnancy and Assessment of Their Determinants," The Journal of Sexual Medicine, Vol. 8, No. 10, 2011, pp. 2859-2867. doi:10.1111/j.1743-6109.2011.02420.x

[21] B. Erol, et al., "A Cross-Sectional Study of Female Sexual Function and Dysfunction during Pregnancy," The Journal of Sexual Medicine, Vol. 4, No. 5, 2007, pp. 13811387. doi:10.1111/j.1743-6109.2007.00559.x

[22] A. P. Leite, et al., "Prevalence of Sexual Dysfunction during Pregnancy," Revista da Associação Médica Brasileira, Vol. 55, No. 5, 2009, pp. 563-568. doi:10.1590/S0104-42302009000500020

[23] L. M. Naldoni, et al., "Evaluation of Sexual Function in Brazilian Pregnant Women,” Journal of Sex \& Marital Therapy, Vol. 37, No. 2, 2011, pp. 116-129. doi:10.1080/0092623X.2011.560537

[24] R. N. Pauls, J. A. Occhino and V. L. Dryfhout, "Effects of Pregnancy on Female Sexual Function and Body Image: A Prospective Study,” The Journal of Sexual Medicine, Vol. 5, No. 8, 2008, pp. 1915-1922. doi:10.1111/j.1743-6109.2008.00884.x
[25] C. Sacomori and F. L. Cardoso, "Sexual Initiative and Intercourse Behavior during Pregnancy among Brazilian Women: A Retrospective Study,” Journal of Sex \& Marital Therapy, Vol. 36, No. 2, 2010, pp. 124-136. doi:10.1080/00926230903554503

[26] E. O. Orji, I. O. Ogunlola and O. B. Fasubaa, "Sexuality among Pregnant Women in South West Nigeria,” Journal of Obstetrics and Gynaecology, Vol. 22, No. 2, 2002, pp. 166-168. doi:10.1080/01443610120113319

[27] F. A. Bello, et al., "Effect of Pregnancy and Childbirth on Sexuality of Women in Ibadan, Nigeria," ISRN Obstetrics and Gynecology, Vol. 2011, 2011, Article ID: 856586.

[28] M. A. DeJudicibus and M. P. McCabe, "Psychological Factors and the Sexuality of Pregnant and Postpartum Women,” The Journal of Sex Research, Vol. 39, No. 2, 2002, pp. 94-103. doi:10.1080/00224490209552128

[29] S. Gokyildiz and N. K. Beji, "The Effects of Pregnancy on Sexual Life,” Journal of Sex \& Marital Therapy, Vol. 31, No. 3, 2005, pp. 201-215. doi:10.1080/00926230590513410

[30] W. H. Masters and V. E. Johnson, "The Sexual Response of the Human Male. I. Gross Anatomic Considerations,” Western Journal of Surgery, Obstetrics, and Gynecology, Vol. 71, 1963, pp. 85-95.

[31] M. Serati, et al., "Female Sexual Function during Pregnancy and after Childbirth,” The Journal of Sexual Medicine, Vol. 7, No. 8, 2010, pp. 2782-2790. doi:10.1111/j.1743-6109.2010.01893.x

[32] R. Basson, "Human Sex-Response Cycles,” Journal of Sex \& Marital Therapy, Vol. 27, No. 1, 2001, pp. 33-43. doi:10.1080/00926230152035831

[33] S. R. Davis, et al., "Circulating Androgen Levels and Self-Reported Sexual Function in Women," Journal of the American Medical Association, Vol. 294, No. 1, 2005, pp. 91-96. doi:10.1001/jama.294.1.91

[34] C. E. Johnson, "Sexual Health during Pregnancy and the Postpartum,” The Journal of Sexual Medicine, Vol. 8, No. 5, 2011, pp. 1267-1284. doi:10.1111/j.1743-6109.2011.02223.x

[35] R. Basson, "Women’s Sexual Dysfunction: Revised and Expanded Definitions,” Canadian Medical Association Journal, Vol. 172, No. 10, 2005, pp. 1327-1333. doi:10.1503/cmaj.1020174

[36] E. A. McClintock, "Handsome Wants as Handsome Does: Physical Attractiveness and Gender Differences in Revealed Sexual Preferences,” Biodemography and Social Biology, Vol. 57, No. 2, 2011, pp. 221-257. doi:10.1080/19485565.2011.615172

[37] S. Wannakosit and V. Phupong, "Sexual Behavior in Pregnancy: Comparing between Sexual Education Group and Nonsexual Education Group," The Journal of Sexual Medicine, Vol. 7, No. 10, 2010, pp. 3434-3438. doi:10.1111/j.1743-6109.2010.01715.x

[38] S. Kitzinger, "Sex before and after Childbirth," Midwife Health Visitor, Vol. 8, No. 9, 1972, pp. 315-318.

[39] K. von Sydow, "Sexuality during Pregnancy and after Childbirth: A Metacontent Analysis of 59 Studies,” Journal of Psychosomatic Research, Vol. 47, No. 1, 1999, pp. 
27-49. doi:10.1016/S0022-3999(98)00106-8

[40] R. Basson, et al., "Report of the International Consensus Development Conference on Female Sexual Dysfunction: Definitions and Classifications,” Journal of Urology, Vol. 163, No. 3, 2000, pp. 888-893. doi:10.1016/S0022-5347(05)67828-7

[41] V. Polomeno, "Sex and Pregnancy: A Perinatal Educator's Guide,” The Journal of Perinatal Education, Vol. 9, No. 4, 2000, pp. 15-27. doi:10.1624/105812400X87879
[42] S. A. Kingsberg, et al., "A Comprehensive Approach to Enhancing Sexual Health Education in the Case Western Reserve University School of Medicine,” International Journal of Impotence Research, Vol. 15, No. S3, 2003, pp. S51-S57. doi:10.1038/sj.ijir.3901073

[43] C. Marwick, "Survey Says Patients Expect Little Physician Help on Sex,” Journal of the American Medical Association, Vol. 281, No. 23, 1999, pp. 2173-2174. 